Meta

Journal des traducteurs

Translators' Journal

\title{
Théorie du sens et sociocritique en traduction littéraire
}

\section{Matthew O. Iwuchukwu}

Volume 55, numéro 3, septembre 2010

URI : https://id.erudit.org/iderudit/045075ar

DOI : https://doi.org/10.7202/045075ar

Aller au sommaire du numéro

\section{Éditeur(s)}

Les Presses de l'Université de Montréal

ISSN

0026-0452 (imprimé)

1492-1421 (numérique)

Découvrir la revue

Citer cet article

Iwuchukwu, M. O. (2010). Théorie du sens et sociocritique en traduction littéraire. Meta, 55(3), 529-544. https://doi.org/10.7202/045075ar

\section{Résumé de l'article}

Le présent article porte sur la théorie et la méthodologie de la traduction, plus précisément sur une alliance originale de la théorie du sens et de la sociocritique en traduction littéraire. Il établit ainsi le lien de complémentarité qui s'impose entre la théorie du sens et la sociocritique dans le processus interprétatif. L'étude soutient que, pour compenser les lacunes des approches linguistique, sémiotique et comparative, le traducteur littéraire doit non seulement faire preuve d'une maîtrise des deux langues (ici l'anglais et le français) et des deux cultures en présence, mais aussi s'armer de principes théoriques qui relèvent à la fois de l'approche interprétative de la traduction et de la sociocritique. Grâce à une analyse du processus interprétatif et à des exemples tirés principalement d'oeuvres littéraires africaines de langue anglaise ou française, elle met en lumière la complémentarité des fonctions interprétative et sociocritique du traducteur. La démarche aide à mieux intégrer dans le processus traductionnel les éléments cognitifs et affectifs fondant la théorie du sens ainsi que les connaissances extralinguistiques sollicitées par la sociocritique. De toute évidence, elle facilite le travail du traducteur littéraire dans la mesure où elle lui permet de réexprimer avec fidélité le vouloir dire original de l'auteur.
Ce document est protégé par la loi sur le droit d'auteur. L'utilisation des services d'Érudit (y compris la reproduction) est assujettie à sa politique d'utilisation que vous pouvez consulter en ligne.

https://apropos.erudit.org/fr/usagers/politique-dutilisation/ 


\title{
Théorie du sens et sociocritique en traduction littéraire
}

\author{
MATTHEW O. IWUCHUKWU \\ University of Nigeria, Nsukka, Nigeria \\ matthew.iwuchukwu@unn.edu.ng
}

\begin{abstract}
RÉSUMÉ
Le présent article porte sur la théorie et la méthodologie de la traduction, plus précisément sur une alliance originale de la théorie du sens et de la sociocritique en traduction littéraire. Il établit ainsi le lien de complémentarité qui s'impose entre la théorie du sens et la sociocritique dans le processus interprétatif. L'étude soutient que, pour compenser les lacunes des approches linguistique, sémiotique et comparative, le traducteur littéraire doit non seulement faire preuve d'une maîtrise des deux langues (ici l'anglais et le français) et des deux cultures en présence, mais aussi s'armer de principes théoriques qui relèvent à la fois de l'approche interprétative de la traduction et de la sociocritique. Grâce à une analyse du processus interprétatif et à des exemples tirés principalement d'œuvres littéraires africaines de langue anglaise ou française, elle met en lumière la complémentarité des fonctions interprétative et sociocritique du traducteur. La démarche aide à mieux intégrer dans le processus traductionnel les éléments cognitifs et affectifs fondant la théorie du sens ainsi que les connaissances extralinguistiques sollicitées par la sociocritique. De toute évidence, elle facilite le travail du traducteur littéraire dans la mesure où elle lui permet de réexprimer avec fidélité le vouloir dire original de l'auteur.
\end{abstract}

\section{ABSTRACT}

This article focuses on the theory and methodology of translation with particular reference to the original alliance between the theory of meaning and sociocriticism in literary translation. Translating literary texts from English into French and vice versa has often posed the problem of inadequate re-expression of a written message, mainly due to the inadequacies of linguistic, semiotic and comparative approaches. Through an analytical study of the literary translation process, the article shows that apart from the need for mastery of two different languages (here English and French) as well as two different cultures involved in the translation process, the literary translator should be conversant with both the interpretive methodology and sociocriticism as an effective means of conveying the writer's message from the source language to the target language. The article seeks to highlight the need for integration of the extra-linguistic components of sociocriticism into the interpretive process, with a number of practical examples drawn mainly from the African literary production in English or French.

\section{MOTS-CLÉS/KEYWORDS}

traduction littéraire, théorie du sens, sociocritique, processus interprétatif literary translation, theory of meaning, sociocriticism, interpretive process

\section{Introduction}

En ce début du troisième millénaire où la mondialisation résonne en écho, les besoins en matière de traduction sont de plus en plus forts. Au Nigeria, par exemple, la traduction vers le français de textes, littéraires ou non, ne cesse de s'intensifier. Ce phénomène peut s'expliquer, d'une part, par le fait que le français se manifeste en 
tant que deuxième langue officielle et, d'autre part, par l'augmentation significative de la production de textes pragmatiques, scientifiques, littéraires et paralittéraires. Ainsi, la littérature igbo compte aujourd'hui plusieurs centaines d'œuvres, publiées également en anglais, dont certaines ont déjà été traduites en français, en particulier celles de romanciers tels que Chinua Achebe, Cyprian Ekwensi et Flora Nwapa (Iwuchukwu 2005: 521-524). Au Nigeria, et de manière générale en Afrique, il existe actuellement des milliers d'œuvres littéraires, tous genres confondus, qui méritent aussi d'être traduites de l'anglais vers le français et vice versa.

Dans ce contexte, nous nous interrogeons sur les principes théoriques et les éléments méthodologiques à mobiliser. Le cadre principal étant celui de la théorie du sens, ou théorie interprétative de la traduction (Lederer 1987: 11-17), l'apport de la sociocritique au processus interprétatif est illustré par la traduction anglais-français d'œuvres de la littérature africaine. Nous interrogeant sur la complémentarité de la théorie du sens et de la sociocritique, nous illustrerons certains concepts et procédés techniques par des références à des œuvres littéraires africaines de langue anglaise ou française.

\section{Les textes littéraires: discursivité et communicabilité de l'écriture}

Nous entendons par «textes littéraires» les différents genres de la littérature écrite, comme le roman, la nouvelle, la poésie et le théâtre. Quel que soit le genre du texte, le langage littéraire se distingue fondamentalement des autres par l'expression subjective de l'émotion de l'écrivain ou de ses personnages, par opposition au langage pragmatique, scientifique ou technologique, doté d'un caractère plus ou moins universel. Comme le soulignent Wellek et Warren, le langage scientifique «tend vers un système de signifiants du type des mathématiques ou de la logique symbolique», alors que le langage littéraire,

fortement connotatif, est loin d'être uniquement référentiel. Il a un côté expressif; il exprime le ton et l'attitude de celui qui parle ou écrit. Et ce langage ne se contente pas d'énoncer et d'exprimer ce qu'il dit; il prétend aussi influer sur l'attitude du lecteur, le persuader, et en définitive le modifier [...]. Des œuvres littéraires diverses s'écartent à des degrés différents du langage scientifique (Wellek et Warren 1971: 32).

Flamand (1983: 119) tient un discours similaire et met en relief la liberté de l'écrivain: «Plus on s'éloigne de la littérature d'art pour se rapprocher des écrits pragmatiques, plus diminue la part de subjectivité. [...] On peut dire que l'on passe d'un langage connotatif, symbolique et allégorique à un langage plutôt dénotatif, descriptif et informatif». À l'opposé du langage scientifique ou pragmatique, l'expressivité et la subjectivité du langage littéraire sont inhérentes à la liberté d'expression artistique dont jouit l'écrivain. Ainsi, dans la préface au roman africain 15 ans, ça suffit! (1985), d'Amadou Ousmane ${ }^{1}$, Idé Oumarou évoque le style particulier utilisé pour dépeindre Siddi Balima, un député corrompu:

15 ans, ça suffit! est peut-être un procès manqué [...]. Je sais qu'ils furent nombreux ceux qui, par opportunisme, par égoïsme, par ruse ou par complicité, profitèrent de la calamité pour s'en mettre plein les poches. Se construire des villas, se faire immatriculer des taxis [...], s'acquérir de redoutables et inestimables relations. Dans ce livre d'Amadou Ousmane, Siddi Balima est leur symbole. Il paiera donc pour tous. Et Amadou Ousmane nous dit tout cela sur un ton tantôt badin, tantôt passionné. C'est 
peut-être sa façon à lui d'extérioriser une indignation longtemps contenue (Oumarou 1985: 7; nous soulignons).

Oumarou souligne l'association signifiante de la forme et du fond, ce qui demeure d'ailleurs une caractéristique du roman en général, qu'il soit africain ou non. Vers la fin du roman, Siddi Balima sera effectivement jugé coupable d'un détournement abusif des deniers de l'État. D'après la longue plaidoirie de l'avocat de la défense, Maître Ali,

(1) il [Siddi Balima] a docilement servi les intérêts de ses maîtres. [...] Nous savons qu'il n'est ni le seul coupable, ni même le seul responsable. La faute vient du système. Du système dans son ensemble. Car la corruption, les détournements abusifs des deniers de l'État [...] les tortures, les exactions en tous genres et j'en oublie, tout cela traduit une seule et cruelle réalité: le peuple est exploité, opprimé, et tous ses droits bafoués.

(Ousmane 1985: 121-122)

La culpabilité de Siddi Balima trahit donc celle du système politique corrompu en place au Bentota, pays sahélien fictif. Ce personnage est devenu le bouc émissaire même du régime politique au pouvoir depuis quinze ans. Le discours juridique du Maître Ali, passionné, a su provoquer les émotions et sensations désirées chez son audience nombreuse au palais de justice. Comme le rapporte le narrateur omniscient extradiégétique du roman,

(2) l'avocat ne se contrôlait plus. Les juges en étaient ébahis, l'assistance médusée. Le Président tenta vainement de le faire taire, mais l'avocat déchaîné débitait son discours avec une passion qu'on ne lui connaissait pas.

(Ousmane 1985: 122)

Le discours de l'avocat et celui de la narration soulignent certes la discursivité et la communicabilité du roman en tant que genre littéraire. Mais il est important de préciser que, dans le cadre de la littérature francophone africaine, le discours romanesque renvoie généralement à des discours déjà tenus dans une société de référence donnée. De manière générale, il s'agit de discours qui, comme nous l'avons signalé dans une étude publiée antérieurement, "se déploient dans tous les domaines du social: discours philosophique, discours politique, discours journalistique, discours religieux, discours onirique et ainsi de suite» (Iwuchukwu 2003a: 303).

En d'autres termes, le discours romanesque fait vraisemblablement partie intégrante du réel discursif emprunté à la société de référence, d'où l'assertion de Labou Tansi dans l'avertissement précédant son roman, L'État honteux (1981) : «Le roman est, paraît-il, une œuvre d'imagination. Il faut pourtant que cette imagination trouve sa place quelque part dans la réalité. J'écris, ou je crie, un peu pour forcer le monde à venir au monde» (Labou Tansi 1981 : 5). La plupart du temps, toute pratique d'écriture (ou pratique discursive) en Afrique trouve sa place dans la réalité africaine dont s'inspire toujours l'écrivain autochtone. Elle permet en outre à celui-ci de transmettre un message en rapport avec le développement national ou régional à un vaste lectorat, notamment africain. Or, c'est bel et bien le message ou le vouloir dire de l'auteur qui fait l'objet même de la traduction littéraire, comme nous le verrons plus loin. Par conséquent, la littérature s'inscrit à la fois dans la dimension de subjectivité de l'auteur et dans une réalité sociale, constatation cruciale pour envisager les approches utiles à la traduction littéraire. 


\section{Traduction littéraire, théorie du sens et sociocritique}

C'est ainsi qu'on peut se poser la question des relations que la traduction littéraire entretient avec la théorie du sens (théorie interprétative de la traduction) et la sociocritique. La mise en place de la théorie du sens a ouvert la voie à l'intégration de la composante culturelle du discours comme élément essentiel de l'opération traduisante. Conçue et développée par Seleskovitch (1986) et d'autres chercheurs à l'École supérieure d'interprètes et de traducteurs (ESIT), à Paris, notamment Lederer et Israël, cette approche s'oppose à la rigidité du transcodage (par ex., Lederer 1986b ; Israël 1990). La théorie du sens puise ses racines dans le champ de l'interprétation de conférence, en particulier dans celui de la traduction orale et simultanée. Sans nul doute, son développement dans le cadre de la traduction a été favorisé par l'évolution du champ des lettres et sciences humaines. La théorie interprétative de la traduction vise à intégrer dans le processus traductionnel ses composantes cognitives et affectives, lesquelles, selon nous, ne vont pas sans faire appel à la sociocritique.

Par ailleurs, en matière de critique littéraire proprement dite, les critiques à l'égard des approches linguistique, structuraliste et sémiotique ont fait naître au début des années 1970 la théorie sociocritique:

La "sociocritique", mot créé par Claude Duchet en 1971, poursuit l'ancienne quête d'une théorie des médiations du social. Loin des théories du "reflet", elle se caractérise par une tension féconde, mais problématique. [...] Travaillant sur les textes dans leurs déterminations sociales et historiques, elle ne veut ni subsumer l'esthétique et la littérarité sous des fonctions sociales positives, ni fétichiser le littéraire comme étant d'une essence à part. En maintenant la tension ou la problématique de l'esthétique et du social, elle se démarque à la fois des approches purement formelles (ou herméneutiques, déconstructionnistes, etc.) du texte littéraire et des approches purement contextuelles, institutionnelles, déterministes (Robin et Angenot 1997: 408).

Autrement dit, dans la perspective de la sociocritique de Dutchet, la démarche du lecteur consiste à mettre en évidence le lien qui s'impose entre le texte littéraire et sa société de référence, entre une pratique d'écriture (ou pratique discursive) et une pratique sociale, entre l'esthétique et le social, entre la réalité et l'imaginaire discursif. Bref, par une contextualisation sociohistorique du texte littéraire, le lecteur sociocritique tend à rendre compte de la prégnance du social dans les modes de discours transposés dans le récit, sans oublier les conditions sociales et culturelles de leur textualisation.

De son côté, Jauss insiste sur le rapport que la littérature entretient avec le modelage de la société: "activité de communication, la littérature n'est pas un simple produit, mais aussi un facteur de production de la société. Elle véhicule des valeurs esthétiques, éthiques, sociales, qui peuvent contribuer aussi bien à transformer la société qu'à la perpétuer telle qu'elle est» (Jauss 1978: 78). Doté d'une valeur communicative, le texte littéraire à traduire évoque donc un monde qui relève autant de l'imaginaire que de la culture.

Du côté de la traductologie, et plus spécifiquement de la théorie du sens, Israël souligne le caractère d'appropriation inhérent à la pratique de la théorie littéraire, caractère imposé, précisément, par les «valeurs culturelles et affectives » du mot, qui font écho à cette alliance de l'imaginaire et du culturel: 
Le traducteur littéraire fait toujours autre chose puisqu'il y a transgression de la lettre, déplacement, écart, autrement dit, appropriation. [...] Toute traduction est appropriation, bonne et mauvaise. [...] Le plus souvent, l'appropriation n'est pas un choix: elle est imposée par la nature même de l'écriture littéraire. Les mots d'abord qui, en apparence, sont ceux de tous les jours, mais qui, chargés de valeurs culturelles et affectives, assument volontiers une fonction symbolique, métaphorique, et s'appellent, se répondent et s'organisent en réseaux (Israël 1991: 18).

L'appropriation, bonne et mauvaise, implique nécessairement une certaine dose de liberté pour le traducteur: liberté du traducteur professionnel qui, même s'il fait autre chose, tend toujours vers la fidélité. La liberté et la fidélité travaillent toujours ensemble dans le processus de la traduction interprétative. Or, la notion de fidélité est plutôt ambivalente en ce sens qu'elle se prête à deux interprétations antithétiques chez les traductologues: "Si pour certains, traduire fidèlement signifie rendre l'expression même du texte original, pour d'autres, une traduction fidèle est celle qui se libère de l'expression pour serrer de plus près l'intention de l'auteur, le message du texte» (St-Pierre 1990: 124).

Pour nous, la fidélité renvoie au respect du vouloir dire de l'auteur repérable dans le message du texte original. Il s'agit donc de la fidélité au sens du discours, le sens étant défini comme "un ensemble déverbalisé, retenu en association avec des connaissances extralinguistiques [... qui] accompagne toujours la perception des signes linguistiques» (Lederer 1994: 240). Comme l'expose Hurtado Albir,

pour être fidèle au sens, le traducteur doit être fidèle d'abord au "vouloir dire" de l'auteur (l'origine préverbale, de tout processus d'expression, la genèse du sens) et, ensuite, dans sa réexpression; il doit être fidèle aux moyens propres qu'offre la langue d'arrivée pour exprimer ce vouloir dire ainsi qu'au destinataire de la traduction pensant à ce que celui-ci peut comprendre ou ne pas comprendre, afin qu'il soit en mesure de saisir le même sens que le destinataire du texte original. Ces trois principes de fidélité [...] sont nécessaires et indissociables si l'on veut être fidèle au sens (Hurtado Albir 1990: 79).

Or, l'on sait que réexprimer avec fidélité le vouloir dire de l'auteur dans la langue d'arrivée (LA) est une tâche complexe, car, comme le souligne Sartre,

dès le départ, le sens n'est plus contenu dans les mots puisque c'est lui, au contraire, qui permet de comprendre la signification de chacun d'eux: et l'objet littéraire quoiqu'il se réalise à travers le langage, n'est jamais donné dans le langage [...] les cent mille mots alignés dans un livre peuvent être lus un à un sans que le sens de l'œuvre en jaillisse; le sens n'est pas la somme des mots; il en est la totalité organique (Sartre 1985: 50-51).

Le sens représente le message véhiculé par les mots, les phrases et l'ensemble des expressions, qui renvoient au vouloir dire original de l'auteur. Le sens saisi dans la LD permet au traducteur littéraire de le rendre avec fidélité dans la LA, en restituant dans celle-ci les sensations, les émotions et les idées exprimées dans le texte original. Mais pour saisir le vouloir dire, le discours littéraire doit être situé dans son contexte socioculturel propre: le texte littéraire, comme le précise Duchet, représente

un discours déjà tenu, parole proférée dans le hors-texte en quelque sorte, dans le passé du texte soit par le on d'une collectivité précise ou indécidable, ou celui des lieux et objets culturels ou symboliques qui portent inscrit en eux le langage de la société qui les a pris en charge (Duchet 1976: 145). 
De manière générale, il s'agit du «discours social» que l'écrivain transforme à sa guise en le transposant dans son texte littéraire. Selon les sociocritiques Robin et Angenot:

Le réel est déjà thématisé, représenté, interprété, sémiotisé dans des discours, des langages, des symboles, des formes culturelles. (Ces discours et langages faisant aussi bien partie du réel). Pour nous, l'écrivain est d'abord quelqu'un qui écoute du point où il se situe dans la société, l'immense rumeur fragmentée qui figure, commente, conjecture, antagonise le monde, cette rumeur, c'est ce qu'en un premier temps nous pourrions appeler discours social (Robin et Angenot 1985: 54).

C'est en cela que le sens fait l'objet à la fois de la traduction interprétative et de la sociocritique. Aussi, nous plaidons pour que le traducteur littéraire professionnel, en plus de maîtriser les deux langues et les deux cultures en présence se manifestant dans l'exercice interprétatif, se dote d'une bonne connaissance de l'orientation théorique et méthodologique de la critique littéraire dans le cadre de la sociocritique.

\section{Le processus interprétatif: le traducteur littéraire comme sociocritique}

Lederer envisage le rôle du traducteur «à un point de jonction» situé à mi-parcours entre l'écrivain et le lecteur:

Le traducteur, tantôt lecteur pour comprendre, tantôt écrivain pour faire comprendre le vouloir dire initial, sait fort bien qu'il ne traduit pas une langue en une autre, mais qu'il comprend une parole et qu'il la transmet à son tour en l'exprimant de manière qu'elle soit comprise. C'est la beauté, c'est l'intérêt de la traduction d'être à ce point de jonction où le vouloir dire de l'écrivain rejoint le vouloir comprendre du lecteur (Lederer 1986b : 19).

En effet, dans la théorie du sens, la traduction est conçue comme «un processus de compréhension et de génération de textes (oraux ou écrits); ce processus est décortiqué en trois phases: compréhension-déverbalisation-réexpression » (Hurtado Albir 1990: 77).

Que faut-il entendre par compréhension dans le cadre de la traduction littéraire? Pour dégager le vouloir dire initial de l'auteur, il faut, selon nous, analyser le discours dans les perspectives certes de la théorie du sens, mais aussi de la sociocritique. Comme le vouloir dire n'est pas nécessairement donné, même dans les connotations du texte littéraire, il faut analyser les «conditions d'émergence, de production et de circulation» du texte ou du discours, qui se trouvent illustrées par les études appropriées dans les champs de l'analyse du discours et de la sociocritique (Rosier 2005: 15). Une partie du sens se situe en effet en dehors du texte dans la mesure où le processus de compréhension est facilité par une contextualisation cognitive et sociohistorique du discours littéraire, réalisée par le lecteur-traducteur. Selon Lederer, le contexte cognitif,

en faisant apparaître l'unité de sens, est tout aussi important pour l'univocité de l'information que l'est le contexte verbal pour l'univocité des mots et des traits sémantiques. Le contexte cognitif est l'ensemble dynamique des informations qu'apporte à l'auditeur le déroulement du discours ou au lecteur celui de la lecture. Égal à zéro aux premiers mots du discours, le contexte cognitif gonfle de plus en plus au fil de l'énonciation. [...] Le contexte cognitif ne diffère de l'ensemble du bagage cognitif que par sa 
durée. On retient suffisamment longtemps en mémoire cognitive ce qu'on vient de lire ou d'entendre pour comprendre la suite du texte ou du discours (Lederer 1986b: 45).

Ainsi, le bagage cognitif et les connaissances extralinguistiques dont dispose le lecteur-traducteur lui permettent de construire des «unités de sens [qui] ne sont pas mesurables quantitativement» à travers des «fragments d'énoncés plus ou moins longs» (Lederer 1986a: 14). Ces unités de sens s'ajoutent à ce que l'on aura déjà retenu au cours de la lecture pour former un sens global. Rappelons que

le lecteur averti sait lire entre les lignes, en misant sur le poids de son bagage encyclopédique, sur la richesse de ses connaissances linguistiques et extralinguistiques. Il sait établir le lien qui s'impose entre l'implicite et l'explicite, entre littérarité et socialité, entre les modes de discours et les conditions socio-historiques de leur mise en texte [...] Il appartient au lecteur de reconstituer par une analyse sociocritique les fragments de discours repérables dans le texte en vue de leur donner un sens global (Iwuchukwu 2003a: 304-305).

Selon cette perspective, prendre connaissance des textes qui précèdent celui qui est à traduire se révèle indispensable. Ainsi, l'histoire du héros romanesque de L'Enfant noir ${ }^{2}$ de Camara Laye (1953) est poursuivie dans Dramouss ${ }^{3}$, du même auteur (1964). Pour comprendre le sens du discours sur l'espace onirique intercalé dans Dramouss (p. 196-231), il faut remonter à l'histoire du petit serpent totémique dans L'Enfant noir (p. 9-39). Sur le plan mystico-religieux, le serpent se révèle être un objet symbolique aux significations multiples, car il évoque diverses images culturelles ou religieuses.

De même, l'histoire de L'étonnante enfance d'Inotan ${ }^{4}$, d'Anthony O. Biakolo (1980), se poursuit dans le roman suivant, publié en anglais, Inotan and the River Goddess $^{5}$ (1992). L'évocation du premier roman intervient au tout début du second:

(3) Inotan was now four years old. Three years had gone since he miraculously saved Warri from bloodbath. His final role in that ethnic conflict had almost been forgotten. It was in fact acknowledged only by a few. Those few included Mukoro and his fellow warriors. They had seen the one year old Inotan in the midst of their adversaries.

(Biakolo 1992: 1)

L'incipit du deuxième roman se caractérise ainsi par une analepse, qui explique qu'Inotan, héros doté de talents extraordinaires qui frappent l'imagination du lecteur, était âgé d'un an seulement dans le premier roman. Ce flash-back aide par ailleurs à mieux saisir le sens du discours romanesque, en rapport avec le conflit interethnique qui sévit à Warri, la ville nigériane où se déroule l'action.

Qu'en est-il de la deuxième phase du processus interprétatif, la déverbalisation? Elle fait suite au processus de compréhension et consiste à extraire le sens compris à partir du texte original. Au dire de Lederer (1994: 46), si ce phénomène «entraîne la disparition des formes, il n'entraîne ni perte ni erreur d'information ». Si, par exemple, un enseignant africain de français raconte à ses élèves en Afrique l'histoire de sa vie d'étudiant étranger en Europe ou en Amérique du Nord, ceux-ci en garderont en mémoire un souvenir cognitif, alors que les mots et les phrases utilisés pour raconter l'histoire ne tarderont pas à disparaître. Il est donc possible de dissocier le sens saisi de la langue de départ. 
Cependant, le vouloir dire de l'écrivain n'étant pas exprimé de façon explicite dans le texte, il faut souligner l'importance des notions d'explicite et d'implicite dans la théorie interprétative de la traduction, qui renvoient à l'apport du réel au processus de déverbalisation:

Autre fondement de la théorie du sens: la part du réel dans la compréhension de l'énoncé. En effet, dans la communication courante, les mots ne disent pas tout et doivent être interprétés en fonction du contexte situationnel qui seul permet de combler les vides (Israël 1990: 32).

Par conséquent, la déverbalisation ne saurait se réaliser sans une contextualisation socioculturelle du discours littéraire, qui se fonde sur les compléments cognitifs et les connaissances extralinguistiques. C'est là que réside aussi l'intérêt commun du texte, du paratexte et du non-dit dans la perspective sociocritique. Convenons d'abord d'une définition du paratexte. Le «paratexte» ou "péritexte» désigne

[ce] que Gérard Genette définit comme «ce par quoi un texte se fait livre et se propose comme tel à ses lecteurs, et plus généralement au public». En somme, le paratexte est l'ensemble des éléments verbaux (le nom de l'auteur, le titre, la préface, les notes, les épigraphes, les prières d'insérer, la bande-annonce, etc.), ou non verbaux (les illustrations, etc.), qui, tout en se situant hors texte, n'en accompagnent pas moins le texte pour en assurer la présence au monde, la réceptivité et la consommation. Le paratexte est donc, pour reprendre le mot de Jean-Yves Tadié commentant les travaux de Genette, l'un des lieux privilégiés de l'action de l'œuvre sur le lecteur (Kadima-Nzuji 1995: 897898).

Le paratexte comporte en effet des modes de discours particuliers qui permettent au lecteur-traducteur de mieux déverbaliser le texte littéraire à traduire, par exemple le discours titulaire,

le discours préfacier, etc. À la lumière de cette définition, le processus de déverbalisation exige que le traducteur littéraire se documente abondamment non seulement sur les textes à traduire, mais aussi sur les civilisations et les cultures qui s'y rattachent.

La phase de déverbalisation est suivie de la réexpression du sens compris (reverbalisation chez Delisle [1988: 63-66]) dans la langue d'arrivée, qui est la troisième et dernière phase du processus interprétatif. Il s'agit de la réexpression du vouloir dire original dans une autre langue, qui est soumise à deux exigences fondamentales, à savoir cerner l'équivalence cognitive et affective et l'exprimer dans la langue cible:

La traduction interprétative est une traduction par équivalences, la traduction linguistique est une traduction par correspondance [...] La traduction, pour être réussie, doit viser à établir une équivalence globale entre le texte original et le texte traduit [...] L'équivalence cognitive découle de la jonction du sémantisme du texte et des compléments notionnels apportés par le traducteur [...] Comment le traducteur réalise-t-il cette équivalence? On a vu que tout texte appelle une interprétation chez le lecteur intéressé. Le traducteur est plus qu'un lecteur intéressé, il est le lecteur par excellence du texte qu'il traduit (Lederer 1994: 50-55).

Les écueils à éviter dans l'opération sont ce que Jeune (1968: 98-99) appelle «les insuffisances ou les inventions du traducteur», l'intention étant, par l'établissement de l'équivalence cognitive et affective, de produire, pour la traduction «le même effet cognitif et émotif sur ses lecteurs que le texte original sur les siens», le traducteur 
étant à la fois «lecteur du texte original et énonciateur en second du sens qu'il en a dégagé» (Lederer 1987: 12).

Aux trois phases du processus interprétatif s'ajoute une quatrième étape, que Delisle (1984: 82-85) appelle analyse justificative, dans laquelle le traducteur devient le lecteur même de sa propre version. Il s'agit pour lui de vérifier si sa traduction rend bien le vouloir dire original de l'auteur, et si elle produit le même effet que l'original. Ainsi, «ce qui provoque le rire dans une langue doit provoquer le rire dans l'autre» (Lefèvre 2002: 24). Une des clés, pour le traducteur professionnel, est de s'assurer que sa version ne calque pas les formes ou les structures de la langue source.

C’est ainsi que nous avons procédé - compréhension-déverbalisation-réexpression-analyse justificative - pour traduire vers l'anglais l'œuvre de Jacques Roumain, Gouverneurs de la rosée $e^{6}$ (1946), dans le cadre de l'alliance de la théorie du sens et de la sociocritique:

(4) Si l'on est d'un pays, si l'on y est né comme qui dirait natif natal, eh bien, on l'a dans les yeux, la peau, les mains, avec la chevelure de ses arbres, la chair de sa terre, les os de ses pierres, le sang de ses rivières, son ciel, sa saveur, ses hommes et ses femmes; c'est une présence dans le cœur, ineffaçable, comme une fille qu'on aime.

(Roumain 1946: 30)

If one is a citizen of Haiti, if one is born there, the people would say: Native land, in actual fact, is present in the eyes, skin and hands, with the trees as her hair, the soil as her skin, the rocks as her bones, the rivers as her blood, her sky, her taste, her men and women: they are all present in the heart, ineffaceable, like a young girl that a man loves.

(Traduction de l'auteur; citée dans Iwuchukwu 2003b: 89)

Nous avons traduit le passage ci-dessus en fonction du rapport que le texte entretient avec le paratexte et le non-dit du texte, dans la perspective sociocritique. De plus, la liberté du traducteur - laquelle n'est pas en conflit avec la fidélité au sens - évoquée plus haut nous a permis d'ajouter le nom de la société de référence (Haiti) dans la première proposition subordonnée de la phrase introduite par if car, selon nous, ce pays était évoqué de façon implicite dans le texte original en français: Si l'on est d'un pays... : If one is a citizen of Haiti... Nous avons ainsi fait ressortir la technique de personnification de cet «objet culturel» (Haïti) utilisé par le romancier, mais sans suivre les formes et structures de la langue de départ. Voilà pourquoi nous avons rendu la comparaison comme une fille qu'on aime: like a young girl that a man loves. Bref, la restitution du sens saisi dans le texte traduit a été faite en fonction des idées exprimées dans le texte original et non en fonction des mots utilisés par le romancier.

Dans la même veine de réflexion, une autre série d'exemples porte sur la traduction interprétative de trois titres de romans, The Bottled Leopard ${ }^{7}$ (Ike, 1989), The Palm-Wine Drinkard ${ }^{8}$ (Tutuola, 1952) et L'Enfant noir (Camara, 1953).

Dans le premier cas, The Bottled Leopard, Étienne Galle s'est écarté du titre original de Chukwuemeka Ike, romancier célèbre igbo du Nigeria, probablement afin d'en lever l'ambigüité au bénéfice des lecteurs francophones connaissant peu l'œuvre:

(5) The Bottled Leopard > Fils de Panthère ${ }^{9}$

(Ike 1989, traduit par Galle 2000) 
Que cache le symbolisme du léopard dans le roman, dans la culture de l'auteur et dans celle du public-lecteur de la version française, notamment les Africains? Pour mieux saisir le sens de Bottled Leopard, il faudrait remonter à l'ère de la colonisation étrangère en Afrique, marquée surtout par la rencontre entre l'africanité traditionnelle et la civilisation occidentale, entre la religion traditionnelle africaine, communément appelée fétichisme (ou animisme), et le christianisme, avec l'enseignement du milieu traditionnel africain et celui de l'école «nouvelle» qui s'y rattachent. À l'instar de plusieurs personnages adolescents des romans africains francophones de l'époque coloniale tels que L'Enfant noir de Camara Laye cité plus haut, Un Enfant d'Afrique d'Olympe Bhely-Quenum ${ }^{10}$, Le Fils du fétiche de David Ananou ${ }^{11}$ et L'Aventure ambiguë de Cheikh Hamidou Kane ${ }^{12}$, le héros de Bottled Leopard, Amobi, part dans une quête de nouvelles valeurs culturelles et spirituelles sur lesquelles se bâtira une Afrique moderne. Cette quête de valeurs l'amène de son milieu traditionnel africain à un collège d'enseignement moderne. Oguzie soutient que les activités pédagogiques formatrices du protagoniste d'Ike dans The Potter's Wheel ${ }^{13}$ se poursuit dans Bottled Leopard, et que l'histoire des deux romans se situe aux alentours des années trente et quarante, plus précisément entre 1939 et 1946, marquées surtout par la Deuxième Guerre mondiale et la propagation du christianisme en Afrique coloniale (Oguzie 1991: 76-102).

La figure du léopard dans ce roman, selon nous, trahit la primauté de la spiritualité et de la morale dans la société orale africaine ou l'africanité traditionnelle. Voilà pourquoi Senghor (1958: 7-22) et Bâ (1972: 119-120) ont fait valoir tour à tour les vertus intrinsèques du fétichisme ancestral. Dans la préface aux Nouveaux contes d'Amadou Koumba de Birago Diop ${ }^{14}$, le premier précise:

Pour le Négro-africain, les vivants, les existants sont au centre du monde. [...] Tout l'univers visible et invisible depuis Dieu jusqu'au grain de sable, en passant par les génies, les ancêtres, les animaux, les plantes, les minéraux est composée de vases communicants, de forces vitales solidaires qui émanent toutes de Dieu (Senghor 1958: 15).

En d'autres termes, envisagés sous l'optique de l'africanologie, les ancêtres divins comme l'oncle Nnanyelugo servent généralement d'intermédiaires entre Chineke ou Chukwu (Dieu créateur ou Dieu suprême chez les Igbo) et leurs semblables Africains vivants, sans oublier la participation collective des animaux et d'autres créateurs de Dieu au fonctionnement du «naturalisme cosmologique» africain (Iwuchukwu 1989: 242-250, voir surtout la partie qui porte sur «le mythe, la religion et la morale»). Sur le plan symbolique, l'oncle Nnanyelugo et les autres ancêtres défunts ne sont pas tout à fait morts dans la mesure où ils participent toujours, mais spirituellement, à la vie communautaire des vivants, comme en témoignent de manière particulière la poésie de la Négritude senghorienne, plus précisément les poèmes Prière aux masques et Le totem $^{15}$. Ceci explique la manifestation du syncrétisme religieux dans le discours de Bottled Leopard, notamment chez les personnages d'Ike, plus ou moins convertis au christianisme. Mentionnons, à titre d'exemple, le héros Amobi et le guérisseur traditionnel, Dibia Offia, qui sont à fois chrétiens et fétichistes. D’après l'épisode onirique intercalé dans le récit, Amobi est «tourmenté» par un léopard symbolique, phénomène interprété par Dibia Offia comme une véritable manifestation du pouvoir de léopard hérité de l'oncle Nnanyelugo. Le guérisseur finira par résoudre définitivement le problème spirituel de l'adolescent par une «mise en bouteille» symbolique du pouvoir mystique. Cette opération réussie de la part du guérisseur permettra au 
jeune héros initié, Amobi, de se transformer à sa guise en un léopard symbolique. Ainsi, par son récit «allégorique» sur le léopard, Chukwuemeka Ike a su remonter aux origines du syncrétisme religieux en Afrique actuelle, qui est aux prises avec des problèmes de croissance socioculturelle, spirituelle et économique. Le syncrétisme religieux dépeint dans l'œuvre, croyons-nous, trahit l'acculturation qui se manifeste chez plusieurs personnages, ainsi que la vision binoculaire interculturaliste des Africains pour le développement de leur continent. Il s'agit d'une vision optimiste de la part du romancier. D'où le commentaire d'Ezenwa-Ohaeto:

Amobi is diagnosed to have inherited the leopard powers of his late Uncle Nnanyelugo.

[...] It is possible to read The Bottled Leopard as a childhood allegory in which the character symbolizes the people at a particular moment of their history. Amobi and Nma embody the hopes of their people and they also represent the struggle to construct a sense of stability out of the chaos of modernism (Ezenwa-Ohaeto 1998: 52).

À la lumière de ces réflexions, quelle est la signification de Bottled Leopard dans la culture du lectorat francophone, en particulier les Africains? Est-ce que Fils de panthère rend avec fidélité le vouloir dire initial de Chukwuemeka Ike dans la langue cible (le français)? Nous proposons plutôt trois titres possibles: L'Enfant-Léopard, Fils de Léopard-fétiche et Fils de Léopard ancestral car, selon nous, ils font ressortir davantage le sens symbolique ou mystique du discours romanesque sur l'africanité.

Le traducteur de The Palm-Wine Drinkard, Raymond Queneau, n'a pas suivi non plus le titre initial d'Amos Tutuola:

(6) The Palm-Wine Drinkard >

L'Ivrogne dans la brousse ${ }^{16}$

(Tutuola 1952, traduit par Queneau 1953/1976)

Il a ajouté l'expression "dans la brousse » à sa version française pour mieux situer l'œuvre dans son milieu socioculturel propre, c'est-à-dire dans une campagne africaine plus ou moins vierge. Comme le léopard chez Chukwuemeka Ike, la brousse revêt un sens symbolique dans le discours romanesque sur l'africanité traditionnelle. Essentiellement, elle se veut un lieu sacré où cohabitent les habitants et les esprits autochtones dans le cadre du fétichisme ancestral. Ainsi, dans des circonstances bien déterminées, le héros-narrateur lui-même et les «esprits-magiciens» de l'œuvre peuvent se métamorphoser en des animaux ou objets symboliques désirés (chien, serpent, poisson, crocodile, oiseau, bâton, etc.). À ce sujet, Kesteloot a raison de souligner que

le mérite de Tutuola est, à nos yeux, de transmettre à l'état brut, précisément, tout l'univers animiste de la campagne africaine, peuplé de monstres, de miracles, de métamorphoses et d'enchantements. C'est une littérature populaire traditionnelle qui essaie de passer à l'expression écrite (Kesteloot 1978: 261).

En vérité, le récit d'Amos Tutuola est une espèce de carrefour de l'oral et de l'écrit dans la société orale africaine, à l'ère coloniale. Il représente le lieu de l'exploitation et de la transformation de la tradition orale africaine en objet littéraire, ce qui justifie bel et bien l'ajustement du titre de la version française du roman par l'ajout de «dans la brousse».

Dans le troisième exemple, L'Enfant noir, le traducteur, James Kirkup, a remplacé l'épithète noir utilisée par le romancier guinéen Laye Camara, par l'adjectif African dans la version anglaise: 


\section{(7) L'Enfant noir}

The African Child ${ }^{17}$

(Camara 1953, traduit par Kirkup 1978)

Selon nous, son choix du mot African exprime de façon explicite le contexte sociohistorique et culturel du discours romanesque, étant donné que l'adjectif noir renvoie généralement à l'Afrique subsaharienne et à sa diaspora dans le monde, avec les spécificités socioculturelles qui s'y rattachent. L'histoire de la vie du héros-narrateur, Camara Laye, est comparable à celle d'Amobi dans The Bottled Leopard. Dans sa quête de nouvelles valeurs culturelles et spirituelles, à l'époque coloniale, Laye Camara reçoit à la fois l'enseignement de son univers traditionnel fétichiste et celui des sciences et des nouvelles technologies occidentales. Sur le plan traditionnel, il faut signaler surtout l'enseignement de son père forgeron dans la forge sacrée, où la "poudre d'or» se transforme à merveille en bijoux pour femmes. Cette opération est facilitée par les «visites» et les interventions bénéfiques répétées d'un petit serpent totémique qui sont sollicitées par le forgeron. D'où le commentaire de Dailly (1977: 37): «Camara Laye, en décrivant la communauté villageoise dans L'Enfant noir, administre à ses lecteurs une magistrale leçon de civilisation africaine». Le titre de la version anglaise du roman, The African Child, est donc tout à fait juste.

Ainsi, les trois traducteurs (Étienne Galle, Raymond Queneau et James Kirkup) ont mis en application dans la traduction des titres romanesques, chacun à sa façon - et avec plus ou moins de pertinence -, le procédé d'ajustement proposé par Nida, comme le rappellent Molina et Hurtado Albir:

Nida proposes three types: additions, subtractions and alterations. They are used: 1) to adjust the form of the message to the characteristics of the structures of the target language; 2) to produce semantically equivalent structures; 3 ) to generate the target language; 4 ) to produce an equivalent communication effect (Molina et Hurtado Albir 2002: 502 ; nous soulignons).

Cette technique a également été utilisée dans les versions anglaises ou françaises des œuvres d'autres écrivains africains tels que Chinua Achebe, Wole Soyinka, Mongo Beti, Sembène Ousmane, etc., que nous ne pouvons pas citer ici, faute d'espace.

Le dernier exemple proposé est constitué par les versions françaises des deux poèmes d'Onuora Ossie Enekwe (poète igbo du Nigeria), Before the war et After the war $^{18}$ (1991), traduits par Étienne Galle ${ }^{19}$ (1995):

(8) Before the war

Beast of the jungle

with claws of fire

a spring, a pounce,

a bite, a pool of blood.

In the city,

tongues of acid,

burning faces,

scaring eyes.

A poison spray

in the dead of night

a mating of witches and vultures.

\section{Avant la guerre}

Bêtes de la jungle aux ongles de feu, surgissant bondissant morsure et mare de sang.

Dans la cité, langues d'acide, visages de flammes, regards de braise. Le poison pullule au cour de la nuit les sorcières et les vautours copulent. 
(9) After the war

In the dark city of the dead

lonely streets

dogs bark at crawling shadows.

Over the murmuring stream,

the wind howls its greetings.

Under a bed

in the deserted village,

a bullet-ridden corpse

ripens into bones.

(Enekwe 1991: 460)

\section{Après la guerre}

Dans la sombre cité des morts

par les rues solitaires

les chiens aboient sur les ombres rampantes.

Par-dessus la rivière des murmures

le vent hurle ses saluts.

Sous un lit,

du village désert

un cadavre criblé de balles

mûrit ses os.

(Enekwe 1995: 92, traduit par Étienne Galle)

Dans ce cas, un certain nombre de formulations pouvaient être traduites directement en français, sans avoir recours à une adaptation notable: before the war: avant la guerre; after the war: après la guerre; beasts of the jungle: bettes de la jungle; in the city: dans la cité; tongues of acid: langues d'acide; pool of blood: mare de sang; under a bed: sous un lit. Par contre, d'autres parties des poèmes s'éloignent d'une traduction directe, par exemple: a spring, a pounce: surgissant bondissant; A poison spray: Le poison pullule; ripens into bones: mûrit ses os, etc. En conformité avec les principes interprétatifs et sociocritiques, Étienne Galle a dû recourir à l'adaptation pour traduire le sens compris du poème d'Enekwe dans la LA, sans suivre les formes et structures des vers poétiques dans la LD. En d'autres termes, aux yeux du traducteur interprétatif et du sociocritique, c'est bel et bien le sens global du discours ou le vouloir dire initial de l'auteur qui fait l'objet de la traduction littéraire, mais, comme en témoignent les versions françaises des deux poèmes, les reformulations linguistiques relatives au sens saisi doivent être faites correctement dans la LA.

Il serait intéressant d'ajouter qu'en raison de la spécificité formelle de la poésie, il ne serait pas possible de conserver les rimes dans le cadre de la traduction de la poésie lyrique, par exemple la poésie française de la Pléiade et la poésie senghorienne sur la Négritude.

\section{Conclusion}

L'intérêt du présent article porte sur l'alliance de la théorie du sens et de la sociocritique en traduction littéraire. À partir d'une analyse du processus interprétatif en quatre phases (compréhension-déverbalisation-réexpression-analyse justificative) et de certaines données sociocritiques (texte, paratexte, et le non-dit du texte), l'étude montre que le sens du texte (ou du discours) peut être traité à fois au moyen de la théorie du sens et de la sociocritique. À la lumière d'une analyse justificative de certaines versions françaises ou anglaises d'œuvres tirées principalement de la production littéraire africaine, notamment la production romanesque, elle révèle que l'alliance des deux approches théoriques facilite non seulement la compréhension et la déverbalisation du texte original dans la langue de départ (LD), mais aussi la réexpression du sens compris dans la langue d'arrivée (LA). La démarche du traducteur consiste à intégrer dans le processus traductionnel les équivalents cognitifs et affectifs qui relèvent de l'approche interprétative ainsi que les connaissances extralinguistiques liées au contexte sociohistorique, dont se préoccupe la sociocritique. Le 
traducteur littéraire professionnel aurait tout à gagner à se doter d'une bonne connaissance de ces deux approches, de manière à ce que cette alliance oriente sa pratique du point de vue théorique et méthodologique. Dans cette perspective, l'étude marque le lancement d'un travail de recherche important sur l'alliance des deux théories en traduction littéraire.

\section{NOTES}

1. Ousmane, Amadou (1985): 15 ans, ça suffit! Dakar: Les Nouvelles Éditions Africaines.

2. Camara, Laye (1953): L’Enfant noir. Paris: Librairie Plon.

3. Camara, Laye (1964): Dramouss. Paris: Présence Africaine.

4. Biakolo, Anthony O. (1980): L'Étonnante enfance d'Inotan. Paris: L'Harmattan.

5. Biakolo, Anthony O. (1992): Inotan and the River Goddess. Ibadan: Patriotic African Series.

6. Roumain, Jacques (1946): Gouverneurs de la rosée. Paris: Les Éditeurs Français Réunis.

7. IкE, Chukwuemeka (1989): The Bottled Leopard. Ibadan: University Press.

8. Tutuola, Amos (1952): The Palm-Wine Drinkard. London: Faber and Faber.

9. Ine, Chukwuemeka (2000): Fils de Panthère. (Traduit par Étienne Galle). Paris: Dapper.

10. Bhely-Quenum, Olympe (1970): Un Enfant d'Afrique. Paris: Librairie Larousse.

11. Ananou, David (1971): Le Fils du fétiche, $2^{e}$ édition. Paris: Nouvelles Éditions Latines.

12. Kane, Cheikh Hamidou (1961): L’Aventure ambiguë. Paris: Union Générale d'Éditions.

13. IкE, Chukwuemeka (1981): The Potter's Wheel. Glasgow: Collins/Fontana.

14. Diop, Birago (1958): Nouveaux contes d'Amadou Koumba. Paris: Présence Africaine.

15. SENGHOR, Léopold Sédar (1977): Prière aux masques - Le totem. In: Abiola IRELE, dir. Selected Poems of Léopold Sédar Senghor. Cambridge: University Press, 46-47.

16. Tutuola, Amos (1953/1976): L'Ivrogne dans la brousse. (Traduit par Raymond Queneau) Paris: Gallimard.

17. Camara, Laye (1978): The African Child. (Traduit par James Kirkup) Douglas: Fontana/Collins.

18. Елекwe, Onuora Ossie (1991): Before the War - After the War. The Literary Review. 34(4):460. (Cité dans Bernard Magnier, dir. Poésie d'Afrique au sud du Sahara 1945-1995. Vol. 1 - Une anthologie. Paris: Presses de l'UNESCO, 92).

19. ЕNekwe, Onuora Ossie (1995): Avant la guerre - Après la guerre. (Traduit par Étienne Galle) In: Bernard Magnier, dir. Poésie d'Afrique au sud du Sahara 1945-1995. Vol. 1. Paris: Presses de l'UNESCO, 92.

\section{RÉFÉRENCES}

BÂ, Amadou Hampaté (1972): Aspects de la civilisation africaine. Personne, culture, religion. Paris: Présence Africaine.

DAILlY, Christophe (1977): Vers une ré[é]valuation idéologique de la littérature africaine. Revue de littérature et d'esthétique négro-africaines. 1:31-43.

Delisle, Jean (1984): L’analyse du discours comme méthode de traduction: Théorie et pratique. Ottawa: Presses de l'Université d'Ottawa.

Delisle, Jean (1988): Translation: An Interpretive Approach. (Traduit par Patricia Logan et Monica Creerr). Ottawa: Presses de l'Université d'Ottawa.

Duchet, Claude (1976): Discours social et texte italique dans Madame Bovary. In: Langages de Flaubert, Actes du colloque de London. Paris: Lettres Modernes Minard, 143-161.

Ezenwa-Ohaeto ou Ohaeto, Ezenwa (1998): The Symbolic Concept of Childhood in Chukwuemeka Ike's The Porter's Wheel and The Bottled Leopard. In: Eldred Dorisimi Jones et Marjorie Jones, dir. Childhood in African Literature. Oxford: James Currey Ltd., 44-53.

Flamand, Jacques (1983): Écrire et traduire. Sur la voie de la création. Ottawa: Éditions Vermillon.

Hurtado Albir, Amparo (1990): La fidélité au sens: un nouvel horizon pour la traductologie. In: Marianne LeDERER, dir. Études traductologiques (en hommage à Danica Seleskovitch). Paris: Lettres Modernes Minard, 75-86. 
IsRAËL, Fortunato (1990): Traduction littéraire et théorie du sens. In: Marianne LEDERER, dir. Études traductologiques (en hommage à Danica Seleskovitch). Paris: Lettres Modernes Minard, 29-43.

IsRAËL, Fortunato (1991): La traduction littéraire: l'appropriation du texte. In: Marianne LEDERER et Fortunato ISRAËL, dir. La Liberté en traduction. Actes du colloque international tenu à l'ESIT. Paris: Didier Érudition, 17-41.

IwUchukwu, Matthew O. (1989): L'Espace dans le roman africain francophone des origines à 1987: visions du monde et idéologies. Thèse de doctorat. Université de Sherbrooke. [Microfiches] (1991) Ottawa: Bibliothèque nationale du Canada.

Iwuchukwu, Matthew O. (2003a): Théorie littéraire et sociocritique: pour une médiation théorique et méthodologique. Nsukka Journal of the Humanities. 13:296-312.

Iwuchukwu, Matthew O. (2003b): Agrarian Space and Vision for Sustainable Rural Development: Roumain's "Gouverneurs de la rosée". Africa and World Literature: University of Nigeria Journal of Literary Studies. 3:87-95.

IwUchukwu, Matthew O. (2005) : Francophonie et production littéraire nigériane en traduction française: dialogue des cultures. Neohelicon. 32(2):521-527.

Jauss, Hans Robert (1978): Pour une esthétique de la réception. (Traduit par Claude MaILlard) Paris: Gallimard.

Jeune, Simon (1968): Littérature générale et littérature comparée. Essai d'orientation. Coll. Situation. Vol. 17. Paris: Lettres Modernes Minard.

Kadima-Nzuji, Mukala (1995): Introduction à l'étude du paratexte du roman zaïrois. Cahiers d'études africaines. 140(XXXV-4):897-909.

Kesteloot, Lilyan (1978): Anthologie négro-africaine. Verviers: Les Nouvelles Éditions Marabout.

Labou Tansi, Sony (1981): L'État honteux. Paris: Éditions du Seuil.

Lederer, Marianne (1994): La Traduction aujourd'hui. Le modèle interprétatif. Paris: HachetteLivre.

LEDERER, Marianne (1987): La théorie interprétative de la traduction. Retour à la traduction. Le Français dans le monde. Numéro spécial août-septembre:11-17.

Lederer, Marianne (1986a): Implicite et explicite. In: Danica Seleskovitch et Marianne LEDERER, dir. Interpréter pour traduire. $2^{\mathrm{e}}$ éd. Paris: Didier Érudition, 37-71.

Lederer, Marianne (1986b) : Transcoder ou réexprimer? In: Danica Seleskovitch et Marianne LEDERER, dir. Interpréter pour traduire. $2^{\mathrm{e}}$ éd. Paris: Didier Érudition, 15-36.

Lefèvre, Kim (2002): La traduction littéraire, dialogue métis. Francophonies du Sud. 1:24.

Molina, Lucía et Hurtado Albir, Amparo (2002): Translation Techniques Revisited: A Dynamic and Functionalist Approach. Meta. 47(4):498-512.

Oumarou, Idé (1985): Préface. In: Amadou Ousmane. 15 ans, ça suffit! Dakar: Les Nouvelles Éditions Africaines, 1.

Oguzie, Benaiah E.C. (1991): Art and Social Relevance: A Study of Artistic Vision and Literary Development of Chukwuemeka Ike. Mémoire de maîtrise non publié. Calabar: University of Calabar.

Robin, Régine et Angenot, Marc (1997): La sociologie de la littérature. In: Jean BessièRe, Eva Kushner, Roland Mortier et al., dir. Histoire des poétiques. Paris: Presses Universitaires de France, 395-409.

Robin, Régine et Angenot, Marc (1985): L'inscription du discours social dans le texte littéraire. Sociocriticism. 1:53-82.

Rosier, Lawrence (2005) : Analyse du discours et sociocritique. Littérature. 140(décembre):1429.

SARTre, Jean-Paul (1985): Qu'est-ce que la littérature? Paris: Éditions Gallimard.

Seleskovitch, Danica (1986): Interpréter un discours n'est pas traduire une langue. In: Danica Seleskovitch et Marianne Lederer, dir. Interpréter pour traduire. $2^{\mathrm{e}}$ éd. Paris: Didier Érudition, 104-115. 
544 MetA, LV, 3, 2010

Senghor, Léopold Sédar (1958): Préface. In: Birago DIOP. Nouveaux contes d'Amadou Koumba. Paris: Présence Africaine, 7-22.

St-Pierre, Paul (1990): La traduction: histoire et théorie. In: Monique C. Cormier et Jacques LeTHUillier, dir. Actes du colloque international "La traduction proligère". Meta. 35(1):119125.

Wellek, René et Warren, Austin (1971): La théorie littéraire. Paris: Éditions du Seuil. 